\title{
Boosting SWIPT via Symbol-Level Precoding
}

\author{
Sumit Gautam ${ }^{\dagger}$, Jevgenij Krivochiza ${ }^{\dagger}$, Alireza Haqiqatnejad ${ }^{\dagger}$, Symeon Chatzinotas, Björn Ottersten \\ Interdisciplinary Centre for Security, Reliability and Trust (SnT), University of Luxembourg, Luxembourg. \\ Email: \{sumit.gautam, jevgenij.krivochiza, alireza.haqiqatnejad, symeon.chatzinotas, bjorn.ottersten\}@ uni.lu \\ ${ }^{\dagger}$ These authors have contributed equally to the work.
}

\begin{abstract}
In this paper, we investigate a simultaneous wireless information and power transmission (SWIPT) system, wherein a single multi-antenna transmitter serves multiple single-antenna users which employ the power-splitting (PS) receiver architecture. We formulate a Symbol-Level Precoding (SLP) based transmit power minimization problem dependent on the minimum signalto-interference-plus-noise ratio (SINR) and energy harvesting (EH) thresholds. We solve the corresponding non-negative convex quadratic optimization problem per time frame of transmitted symbols and study the benefits of proposed design under ZeroForcing (ZF) Precoding, Direct Demand SLP (DD-SLP), and Squared-Root Demand SLP (RD-SLP) techniques. A static PSratio is fixed according to the SINR and EH demands to enable the segregation of intended received signals for information decoding (ID) and EH, respectively. Numerical results show the property conservation of SINR-enhancement via SLP at the ID unit while increasing the harvested energy at each of the end-users.
\end{abstract}

Index Terms-MU-MISO, power optimization, SLP, SWIPT.

\section{INTRODUCTION}

The forthcoming generation of wireless communications (5G) and the notion of Internet-of-Things (IoTs) have primarily focused on high throughput with low link latency [1]. Recent developments in the respective fields indicate a compromise between the system performance (or capacity demands) and the power consumption (or battery drainage) at the user nodes due to complex hardware and heavy applications. In this vein, the adoption of power optimization and energy harvesting $(\mathrm{EH})$ methods come in handy [2], [3].

In order to enhance the link throughput, symbol-level precoding (SLP) techniques seem promising [4]-[7]. Taking into consideration the $\mathrm{EH}$ aspect, the power-splitting (PS)-based mechanism for simultaneous wireless information and power transmission (SWIPT) has shown immense potential [8]. In this context, the non-linear characteristic of the EH module is noteworthy [9]. The authors in [10], [11] investigated SLP with SWIPT, however, with consideration of a linear EH operation under a sophisticated system design.

In this paper, we focus on terrestrial communication through a multi-user multiple-input single-output (MU-MISO) channel. We provide three energy efficient SLP-SWIPT strategies to minimize the overall transmit power while maintaining the joint minimal signal-to-interference-plus-noise ratio (SINR) and EH requirements at the receiver terminals. Specifically, the novelty and main contributions of this work are: (i) adoption of simplified SLP-SWIPT model with static PS-based operation (which curbs the optimization of PS factor), (ii) novel approach with joint weighted SINR and EH demand operations enable the investigation of proposed frameworks from a pricing perspective, while their binary selections may facilitate system analysis for explicit (all) users with either SINR or EH specific demands, and (iii) we show that SLP enhances the rate-energy (R-E) trade-off for the SWIPT systems.

The remainder of this paper is organized as follows. In Section II, we describe the system model. We illustrate the three proposed SLP-SWIPT techniques with respective optimization problem formulations and solutions in Section III, Section IV and Section V. Numerical results are shown in Section VI, followed by concluding remarks in Section VII.

Notation: Upper-case and lower-case bold-faced letters are used to denote matrices and column vectors. The superscripts $(\cdot)^{H},(\cdot)^{\dagger}$ and $(\cdot)^{-1}$ represents Hermitian matrix, matrix transpose and inverse operations, respectively. $\|\cdot\|_{2}$ is the Euclidean norm, $|\cdot|$ is an absolute magnitude of a complex value and $\mathbb{E}\{\cdot\}$ denotes the expectation value. The real and imaginary parts of a complex value are defined as $\operatorname{Re}(\cdot)$ and $\operatorname{Im}(\cdot)$. The imaginary unit is denoted as $\iota^{2}=-1$. Square diagonal matrices are denoted as $\operatorname{diag}[\cdot]$ with the elements defined on their main diagonal. The operator $\otimes$ stands for the Kronecker product.

\section{System Model}

The system model considered in this work consists of a single transmitter equipped with $N_{t}$ antennas and a number of receiver terminals $\left(N_{u}\right)$, spread across the coverage area. We assume a full-frequency re-use scenario i.e., the same frequency and time utilization by all the transmit antennas. The precoding technique assists in mitigating the multi-user interference. In the specified MU-MISO channel model, the received signal at the $i$-th terminal is given by $y_{i}=\mathbf{h}_{i}^{\dagger} \mathbf{x}$, where $\mathbf{h}_{i}^{\dagger}$ is a $1 \times N_{t}$ vector representing the complex channel coefficients between the $i$-th user terminal and the $N_{t}$ transmit antennas, $\mathbf{x}$ is defined as the $N_{t} \times 1$ vector of the transmitted symbols at a certain symbol period. Additive White Gaussian Noise (AWGN) is always present in the system, however, in the following section we assume the noise power is negligible. We conduct numerical results against AWGN channel in Section VI. Taking into consideration the whole set of receiver terminals, the linear received signal model is given by

$$
\mathbf{y}=\mathbf{H x}=\mathbf{H W} \text { s, }
$$

where $\mathbf{y}=\left[y_{i} \in \stackrel{\mathbf{C}}{\mathbf{C}} \mathbf{3}, \mathbf{x} \in \mathbb{C}^{N_{t} \times 1}\right.$, and $\mathbf{s} \in \mathbb{C}^{N_{u} \times 1}$ and $\mathbf{H}=\left[\mathbf{h}_{i}^{\dagger} \in \mathbb{C}^{1 \times N_{t}}\right]$, where $i=1, \ldots, N_{u}$. In this scenario, we define a precoding matrix $\mathbf{W} \in \mathbb{C}^{N_{t} \times N_{u}}$ which maps the information symbols $\mathbf{s}$ into precoded symbols $\mathbf{x}$. We consider the data symbols $\mathbf{s}$ to be unit variance complex vectors, $\mathbb{E}\left\{\left|s_{i}\right|^{2}\right\}=1$ for every $i=1,2, \ldots, N_{u}$. Upon its reception, the received signal is split into two fractions according to a PS-mechanism. ${ }^{1}$ Let the signals dedicated to the SINR and EH

\footnotetext{
${ }^{1}$ A static PS process is employed to provide tractability to the system hardware from the following view-points: (i) no additional (dynamic) optimization variable, and (ii) dependence of the PS ratio on the corresponding SINR and $\mathrm{EH}$ demands at the device facilitates in its easy computation and desired signal distribution, while ensuring that the corresponding demands are met.
} 
units of $i$-th user be represented as $y_{\mathrm{ID}_{i}}$ and $y_{\mathrm{EH}_{i}}$, respectively. In order to extract the signal energy at $i$-th user, the sigmoidal function based non-linear EH model [12] is defined as

$$
\mathcal{E}_{i}^{\mathcal{N}}=\frac{\mathcal{E}^{\prime}}{1-\phi} \cdot\left(\frac{1}{1+\exp \left(-\alpha\left|y_{\mathrm{EH}_{i}}\right|^{2}+\alpha \beta\right)}-\phi\right),
$$

where $\phi \triangleq \frac{1}{1+\exp (\alpha \beta)}$, and $\mathcal{E}^{\prime}, \alpha$ and $\beta$ are the constant $\mathrm{EH}$ circuitry parameters. In the following, we develop and analyze the Zero-Forcing (ZF) based SLP-SWIPT framework.

\section{ZERO-FORCING BASED SLP DESIGN FOR SWIPT}

In this section, we intend to develop a basic technique for enabling SWIPT via ZF Precoding design. Let the optimized precoded signal be represented as follows

$$
\mathbf{x}=\mathbf{W}\left(\sqrt{\omega_{R}} \boldsymbol{\Gamma}+\sqrt{\omega_{E}} \boldsymbol{\Xi}\right) \mathbf{s},
$$

where $\Gamma$ is the per terminal minimal SINR requirements (as in [13]) and $\boldsymbol{\Xi}$ is the minimal EH demands respectively, defined as

$$
\text { as } \quad \boldsymbol{\Gamma}=\operatorname{diag}\left[\begin{array}{llll}
\sqrt{\gamma_{1}}, & \sqrt{\gamma_{2}}, & \ldots, & \sqrt{\gamma_{N_{u}}}
\end{array}\right] \text {, }
$$$$
\text { and } \quad \boldsymbol{\Xi}=\operatorname{diag}\left[\sqrt{\xi_{1}}, \quad \sqrt{\xi_{2}}, \ldots, \quad \sqrt{\xi_{N_{u}}}\right] \text {, }
$$

with $\omega_{R}$ and $\omega_{E}$ denoting the corresponding weights ${ }^{2}$ for the SINR and EH demands. Note that $\boldsymbol{\Xi}$ is an up-scaled version of the original linear EH demands. This transformation is necessary to address the non-linearity at the EH module. In simple terms, if $\xi_{i}^{\prime}$ is the linear EH demand by the $i^{\text {th }}$ user, then $\xi_{i}$ is the up-scaled EH demand, computed according to the analysis in Appendix A of [2].

In the following, we split the problem of constructing optimal beamforming vectors into two independent tasks: channel orthogonalization and optimal symbol mapping for energy efficiency. In this case, we define the precoding matrix $(\mathbf{W})$ as the $\mathrm{ZF}$ linear precoder

$$
\mathbf{W}_{\mathrm{ZF}}=\hat{\mathbf{H}}^{H}\left(\hat{\mathbf{H}} \hat{\mathbf{H}}^{H}\right)^{-1} / f_{Z F},
$$

where $f_{Z F}=\sqrt{\sum_{n=1}^{N_{t}} \sum_{m=1}^{N_{u}} \mathbf{W}_{\mathrm{ZF}}^{2} \text { is }}$ is a rescaling factor to account for sum power constraints, $\hat{\mathbf{H}}$ is the channel matrix estimated from the channel state information (CSI). We choose $\mathrm{ZF}$ for its properties to orthogonalize the channel so that under the assumption of the perfect channel estimation $(\hat{\mathbf{H}}=\mathbf{H})$ the SINRs of the received symbols are linearly related to the $\boldsymbol{\Gamma}$ and the EH demand metrics are linearly related to the $\boldsymbol{\Xi}$, applied on the transmitted symbols assuming a noise-free scenario:

$$
\mathbf{y}=\mathbf{H W}_{\mathrm{ZF}}\left(\sqrt{\omega_{R}} \boldsymbol{\Gamma}+\sqrt{\omega_{E}} \boldsymbol{\Xi}\right) \mathbf{s}=\left(\sqrt{\omega_{R}} \boldsymbol{\Gamma}+\sqrt{\omega_{E}} \boldsymbol{\Xi}\right) \mathbf{s} .
$$

In order to represent the PS mechanism at the receiver, we define

$$
\boldsymbol{\rho}=\operatorname{diag}\left[\varrho_{1}, \varrho_{2}, \ldots, \varrho_{N_{u}}\right],
$$

where $\rho$ is per terminal PS ratio, with static value at the $i^{\text {th }}$ user computed as

$$
\varrho_{i}=\frac{\sqrt{\omega_{R} \gamma_{i}}}{\sqrt{\omega_{R} \gamma_{i}}+\sqrt{\omega_{E} \xi_{i}}} .
$$

Following the PS process, signals at the ID and EH modules of the corresponding receivers are respectively defined as

\footnotetext{
${ }^{2}$ The weights corresponding to the SINR and EH terms may be elucidated as a pricing method for SLP-SWIPT systems. Herein, we define $\omega_{R}=0.015$ currency units/dB and $\omega_{E}=1$ currency units/ $\mu \mathrm{J}$ [14]. Alternatively, the binary nature of weights, i.e., either $\omega_{R}=1$ and $\omega_{E}=0$, or vice-versa may be used to study SINR explicit or EH only type of mechanisms, respectively.
}

$$
\begin{aligned}
\mathbf{y}_{\mathrm{ID}} & =\boldsymbol{\rho}\left(\left(\sqrt{\omega_{R}} \boldsymbol{\Gamma}+\sqrt{\omega_{E}} \boldsymbol{\Xi}\right) \mathbf{s}\right), \\
\text { and } \quad \mathbf{y}_{\mathrm{EH}} & =(\mathcal{I}-\boldsymbol{\rho})\left(\left(\sqrt{\omega_{R}} \boldsymbol{\Gamma}+\sqrt{\omega_{E}} \boldsymbol{\Xi}\right) \mathbf{s}\right),
\end{aligned}
$$

where $\mathcal{I}$ is the identity matrix of $N_{u} \times N_{u}$ dimensions. Due to the implementation of ZF-precoder, the computation of $\rho$ is straightforward under an assumption of a noise-free scenario. Such kind of PS-mechanism guarantees that the individual minimum weighted SINR and EH demands will be satisfied. In the subsequent sections, we develop two SLP techniques for further enhancement of the system performance.

\section{Direct Demand SLP Design FOR SWIPT}

In this section, we define an optimization problem of the computationally efficient SLP technique, which aims to minimize the sum power of the precoded symbols at the gateway side. SLP techniques optimally preserve constructive interference in MIMO systems to improve the power efficiency at the transmitter's side and the service availability at the user side. These techniques are guided by the knowledge of CSI and transmitted modulated signals for all the users. Hence, different techniques have to be specifically designed for different types of the waveform. We derive this technique for the $M$-th order quadrature amplitude modulation ( $M$-QAM). It reduces the sum power of the precoded symbols by optimally increasing the amplitudes of the initial data symbols to exploit the constructive interference at the receiver side. The method optimally preserves constructive interference components to decrease the total transmitted power at the transmitter side.

To achieve this, the SLP technique needs to recalculate a perturbation vector $\mathbf{u}=\left[u_{1}, u_{2}, \ldots, u_{i}\right] \in \mathbb{C}^{N_{u} \times 1}$ for a set of symbols $\mathbf{S}$ in every transmission time frame to construct the optimized precoded signal given by

$$
\mathbf{x}=\mathbf{W}_{\mathrm{ZF}}\left(\sqrt{\omega_{R}} \boldsymbol{\Gamma}+\sqrt{\omega_{E}} \boldsymbol{\Xi}\right)(\mathbf{s}+\mathbf{u}),
$$

where $\boldsymbol{\Gamma}$ and $\boldsymbol{\Xi}$ are the corresponding SINR and EH demands, as represented in (4) and (5), respectively.

In the case of $\hat{\mathbf{H}}=\mathbf{H}$, the SINRs of the received symbols are linearly related to $\Gamma$ and the EH demand metrics are linearly related to $\Xi$ :

$$
\begin{aligned}
\mathbf{y} & =\mathbf{H W}_{\mathrm{ZF}}\left(\sqrt{\omega_{R}} \boldsymbol{\Gamma}+\sqrt{\omega_{E}} \boldsymbol{\Xi}\right)(\mathbf{s}+\mathbf{u}) \\
& =\left(\sqrt{\omega_{R}} \boldsymbol{\Gamma}+\sqrt{\omega_{E}} \boldsymbol{\Xi}\right) \mathbf{s}+\left(\sqrt{\omega_{R}} \boldsymbol{\Gamma}+\sqrt{\omega_{E}} \boldsymbol{\Xi}\right) \mathbf{u} .
\end{aligned}
$$

The proposed Direct Demand-based SLP (DD-SLP) approach will build upon the ZF precoder and will improve the energy efficiency by optimizing every transmitted set of symbols. The optimal symbol mapping is derived in [13].

In this regard, we consider that all the symbols are mapped in the first quadrnt of the complex plane by transforming the actual complex data symbols with the equivalent symbols $\tilde{\mathbf{s}} \in$ $\mathbb{C}^{N_{u} \times 1}$, where $\tilde{s}_{i}=b_{i} s_{i}, \operatorname{Re}\left(\tilde{s}_{i}\right) \geq 0$ and $\operatorname{Im}\left(\tilde{s}_{i}\right) \geq 0$ for every $i=1,2, \ldots, N_{u}$. The variable $b_{i}$ represents the rotation of the symbol vectors into the first quadrature of the complex plane and is defined as

$$
b_{i}=\left\{\begin{array}{ll}
1 & \text { if } \operatorname{Re}\left(s_{i}\right)>0 \text { and } \operatorname{Im}\left(s_{i}\right)>0 \\
\iota 1 & \text { if } \operatorname{Re}\left(s_{i}\right)<0 \text { and } \operatorname{Im}\left(s_{i}\right)>0 \\
-\iota 1 & \text { if } \operatorname{Re}\left(s_{i}\right)>0 \text { and } \operatorname{Im}\left(s_{i}\right)<0 \\
-1 & \text { if } \operatorname{Re}\left(s_{i}\right)<0 \text { and } \operatorname{Im}\left(s_{i}\right)<0
\end{array},\right.
$$


for $i=1,2, \ldots, N_{u}$. The following equality is therefore respected

$$
\mathbf{s}=\mathbf{B} \tilde{\mathbf{s}}
$$

where $\mathbf{B}$ is a diagonal matrix, where the rotation vectors $b_{i}$ are its diagonal elements such as:

$$
\mathbf{B}=\operatorname{diag}\left[b_{1}, \quad b_{2}, \quad b_{3}, \ldots, b_{i}\right] .
$$

The four possibilities of symbol positioning $\left(p_{i}, i=1, \ldots, 4\right)$ are identified on the complex plain regardless of the modulation order $M$ considered and the constraints on the perturbation vector based on the position of a symbol are summarized in Table I of [13]. We replace the perturbation vector $\mathbf{u}$ with a new vector $\tilde{\mathbf{u}}=\left[\tilde{u}_{1}, \tilde{u_{2}}, \ldots, \tilde{u}_{i}\right] \in \mathbb{C}^{N_{u} \times 1}$, where $\operatorname{Re}\left(\tilde{u}_{i}\right) \geq 0$ and $\operatorname{Im}\left(\tilde{u}_{i}\right) \geq 0$ for every $i=1,2, \ldots, N_{u}$. Finally, we rewrite the equation (12) as

$$
\mathbf{x}=\mathbf{W}_{\mathrm{ZF}}\left(\sqrt{\omega_{R}} \boldsymbol{\Gamma}+\sqrt{\omega_{E}} \boldsymbol{\Xi}\right) \mathbf{B}(\tilde{\mathbf{s}}+\tilde{\mathbf{u}}) .
$$

We define the optimization problem to minimize the sum power of the precoded symbols vector $\mathbf{x}$ for any $M$-QAM constellation as

$$
\begin{aligned}
& \min _{\mathbf{x}, \tilde{\mathbf{u}}}\left\|_{\mathbf{x}}\right\|_{2} \\
& \text { s. t. } \quad(C 1):\left\{\begin{array}{l}
\operatorname{Re}\left(\tilde{u}_{i}\right) \geq 0 \\
\operatorname{Im}\left(\tilde{u}_{i}\right) \geq 0
\end{array}, \tilde{s}_{i} \in p_{1},\right. \\
&(C 2):\left\{\begin{array}{l}
\operatorname{Re}\left(\tilde{u}_{i}\right) \geq 0 \\
\operatorname{Im}\left(\tilde{u}_{i}\right)=0
\end{array}, \tilde{s}_{i} \in p_{2},\right. \\
&(C 3):\left\{\begin{array}{l}
\operatorname{Re}\left(\tilde{u}_{i}\right)=0 \\
\operatorname{Im}\left(\tilde{u}_{i}\right) \geq 0
\end{array}, \tilde{s}_{i} \in p_{3},\right. \\
&(C 4):\left\{\begin{array}{l}
\operatorname{Re}\left(\tilde{u}_{i}\right)=0 \\
\operatorname{Im}\left(\tilde{u}_{i}\right)=0
\end{array}, \tilde{s}_{i} \in p_{4},\right.
\end{aligned}
$$

for all $i=1,2, \ldots, N_{u}$. By substituting (17) in (18) and by employing suitable transformations, we can obtain and efficiently solve a convex problem (as in [13]). In general, the transformed optimization problem can be solved using CVX tools for MATLAB [15]. At the user terminal, the received signal is split according to the PS ratio defined in (9) and further processing is performed as previously described. In the next section, we present an alternative SLP method to further improve system performance.

\section{SQUARED-Root DEMAND SLP DESIGN FOR SWIPT}

In this section, we develop a SWIPT-enabled power minimization SLP problem with SINR constraints based on the so-called distance-preserving constructive interference (CI) regions introduced in [16]. The design problem aims to minimize the instantaneous (per-symbol) total transmit power while ensuring the corresponding CI constraints as well as given SINR requirements for all the receiver terminals.

In what follows, for the sake of convenience, we define the equivalent real-valued notations: $\overline{\mathbf{x}} \triangleq\left[\operatorname{Re}(\mathbf{x})^{T}, \operatorname{Im}(\mathbf{x})^{T}\right]^{T}$, and for all $i=1, \ldots, N_{u}$, we denote $\mathbf{s}_{i} \triangleq\left[\operatorname{Re}\left(s_{i}\right), \operatorname{Im}\left(s_{i}\right)\right]^{T}$ and

$$
\mathbf{H}_{i} \triangleq\left[\begin{array}{rr}
\operatorname{Re}\left(\mathbf{h}_{i}^{T}\right) & -\operatorname{Im}\left(\mathbf{h}_{i}^{T}\right) \\
\operatorname{Im}\left(\mathbf{h}_{i}^{T}\right) & \operatorname{Re}\left(\mathbf{h}_{i}^{T}\right)
\end{array}\right] .
$$

The distance-preserving CI regions are defined such that any two points belonging to distinct CI regions are distanced by at least the distance between the corresponding constellation points [16]. Denoting by $\mathcal{D}_{i}$ the distance-preserving CI region associated with symbol $\mathbf{s}_{i}$, we can obtain the optimal transmit signal $\mathrm{x}$ by solving the following optimization problem:

$$
\begin{array}{cl}
\min _{\overline{\mathbf{x}}} & \overline{\mathbf{x}}^{T} \overline{\mathbf{x}} \\
\text { s.t. } & \mathbf{H}_{i} \overline{\mathbf{x}} \in \mathcal{D}_{i}, i=1, \ldots, N_{u},
\end{array}
$$

Unlike conventional linear precoding schemes, this nonlinearprecoded signal $\mathbf{x}$ may not be explicitly decomposed as a linear combination of distinct signatures (i.e., precoding vectors). It should further be noted that in formulating (19), we implicitly assume that the downlink channel vectors for single antenna users are perfectly and instantaneously known to the transmitter.

The CI constraints $\mathcal{D}_{i}$ in problem (19), as it has been shown in [5], can be written in an explicit compact form as

$$
\overline{\mathbf{H}} \overline{\mathbf{x}}=\boldsymbol{\Sigma}\left(\sqrt{\omega_{R}} \overline{\boldsymbol{\Gamma}}+\sqrt{\omega_{E}} \overline{\boldsymbol{\Xi}}\right) \overline{\mathbf{s}}+\mathbf{A}^{-1} \boldsymbol{\Theta} \mathbf{t},
$$

where we use the following definitions: $\overline{\mathbf{H}} \triangleq\left[\mathbf{H}_{1}^{T}, \ldots, \mathbf{H}_{N_{u}}^{T}\right]^{T}$; $\mathbf{A} \triangleq \operatorname{blkdiag}\left(\mathbf{A}_{1}, \ldots, \mathbf{A}_{N_{u}}\right) ; \mathbf{A}_{i}=\left[\mathbf{a}_{i, 1}, \mathbf{a}_{i, 2}\right]^{T}$ for any $i \in$ $\left\{1, \ldots, N_{u}\right\}$ is a $2 \times 2$ real-valued matrix containing the normal vectors of the CI boundaries ${ }^{3} ; \boldsymbol{\Sigma} \triangleq \operatorname{diag}\left(\sigma_{1}, \ldots, \sigma_{N_{u}}\right) \otimes \mathbf{I}_{2}$; $\overline{\boldsymbol{\Gamma}} \triangleq \boldsymbol{\Gamma} \otimes \mathbf{I}_{2}$ and $\overline{\boldsymbol{\Xi}} \triangleq \boldsymbol{\Xi} \otimes \mathbf{I}_{2}$ with $\boldsymbol{\Gamma}$ and $\boldsymbol{\Xi}$ as defined in (4) and (5), respectively; $\overline{\mathbf{s}} \triangleq\left[\mathbf{s}_{1}, \ldots, \mathbf{s}_{N_{u}}\right]^{T} ; \boldsymbol{\Theta}$ denotes a diagonal weighting matrix with a diagonal element being one if the corresponding symbol is an outer constellation point ${ }^{4}$ and zero otherwise, i.e.,

$$
\boldsymbol{\Theta} \triangleq \operatorname{diag}\left(w_{1}, \ldots, w_{N_{u}}\right) \otimes \mathbf{I}_{2}, \quad w_{i}= \begin{cases}1, & \mathbf{s}_{i} \text { is outer point } \\ 0, & \mathbf{s}_{i} \text { is inner point. }\end{cases}
$$

Furthermore, the $2 N_{u} \times 1$ non-negative slack vector-variable $\mathbf{t} \triangleq\left[\mathbf{t}_{1}, \ldots, \mathbf{t}_{N_{u}}\right]^{T}$, where $\mathbf{t}_{i}=\left[t_{i, 1}, t_{i, 2}\right]^{T}$, collects the orthogonal distances of the received symbol $\overline{\mathbf{H}}_{i} \overline{\mathbf{x}}$ to its corresponding CI boundaries for all $i=1, \ldots, N_{u}$. For a detailed discussion on the characteristics of distance-preserving regions, the interested readers are referred to [17].

Using (20), the SWIPT-enabled SLP design problem of interest can be formulated as a linearly-constrained quadratic program (LCQP), i.e.,

$$
\begin{aligned}
\min _{\mathbf{x}, \mathbf{t} \succeq \mathbf{0}} & \overline{\mathbf{x}}^{T} \overline{\mathbf{x}} \\
\text { s.t. } & \overline{\mathbf{H}} \overline{\mathbf{x}}=\boldsymbol{\Sigma}\left(\sqrt{\omega_{R}} \overline{\mathbf{\Gamma}}+\sqrt{\omega_{E}} \overline{\boldsymbol{\Xi}}\right) \overline{\mathbf{s}}+\mathbf{A}^{-1} \boldsymbol{\Theta} \mathbf{t},
\end{aligned}
$$

Thereby, the optimal transmit vector $\overline{\mathbf{x}}$ is directly obtained by solving the optimization problem (22) on a symbol-by-symbol basis. It is straightforward to show that the optimal (minimal power) symbol-level precoded transmit signal obtained from the LCQP in (22) has the following structure:

$$
\overline{\mathbf{x}}^{*}=\overline{\mathbf{W}}_{\mathrm{ZF}}\left(\boldsymbol{\Sigma}\left(\sqrt{\omega_{R}} \overline{\boldsymbol{\Gamma}}+\sqrt{\omega_{E}} \overline{\boldsymbol{\Xi}}\right) \overline{\mathbf{s}}+\mathbf{A}^{-1} \boldsymbol{\Theta} \mathbf{t}^{*}\right),
$$

where $\overline{\mathbf{W}}_{\mathrm{ZF}} \triangleq \overline{\mathbf{H}}^{T}\left(\overline{\mathbf{H}} \overline{\mathbf{H}}^{T}\right)^{-1}$ is the equivalent real-valued representation of the zero-forcing precoding matrix in (6) and $\mathbf{t}^{*}$ denotes the optimal solution to the following non-negative least squares (NNLS) problem:

$$
\min _{\mathbf{t} \succeq \mathbf{0}}\left\|\overline{\mathbf{W}}_{\mathrm{ZF}} \boldsymbol{\Sigma}\left(\sqrt{\omega_{R}} \overline{\boldsymbol{\Gamma}}+\sqrt{\omega_{E}} \overline{\boldsymbol{\Xi}}\right) \overline{\mathbf{s}}+\overline{\mathbf{W}}_{\mathrm{ZF}} \mathbf{A}^{-1} \boldsymbol{\Theta} \mathbf{t}\right\|_{2}^{2} .
$$

${ }^{3}$ The sub-matrices $\left\{\mathbf{A}_{1}, \ldots, \mathbf{A}_{N_{u}}\right\}$ are shown to be always full-rank square (hence invertible) matrices, so is $\mathbf{A}$.

${ }^{4}$ Any constellation point residing on the convex hull of the constellation set is referred to as outer constellation point. 


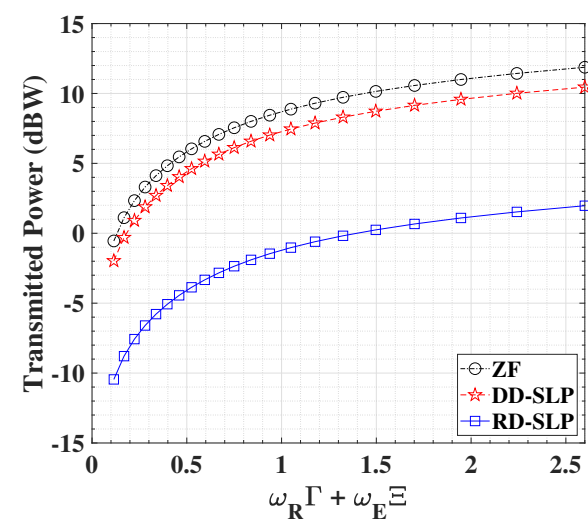

(a)

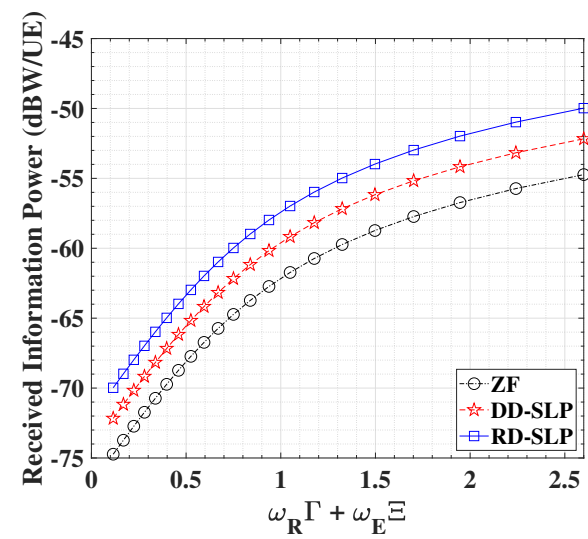

(b)

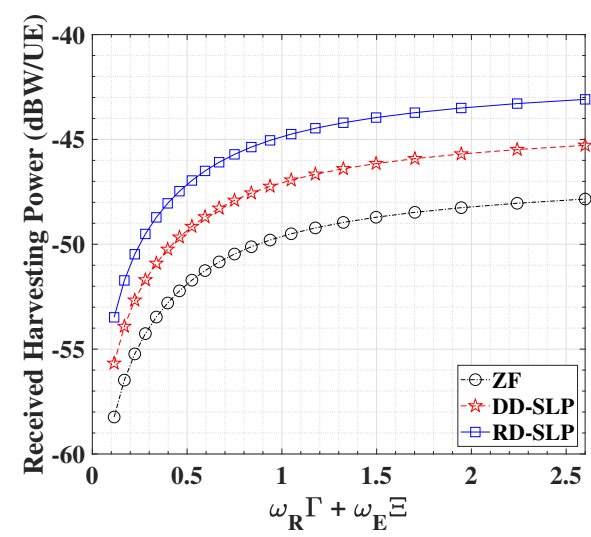

(c)

Fig. 1. Comparison between the ZF, DD-SLP and RD-SLP techniques with $\gamma_{i}=[0: 1: 20] \mathrm{dB}$ and $\xi_{i}=[0.1: 0.05: 1.1] \mu \mathrm{J}$. (a) Transmitted power versus Pricing Metric, (b) Average Received Information Power versus Pricing Metric and (c) Average Received Harvesting Power versus Pricing Metric.

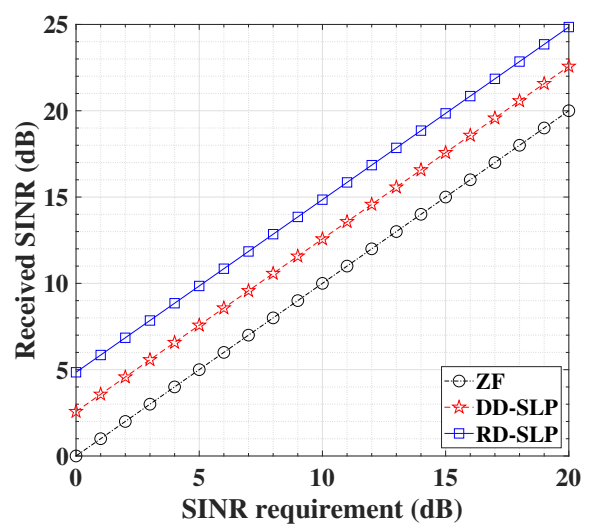

(a)

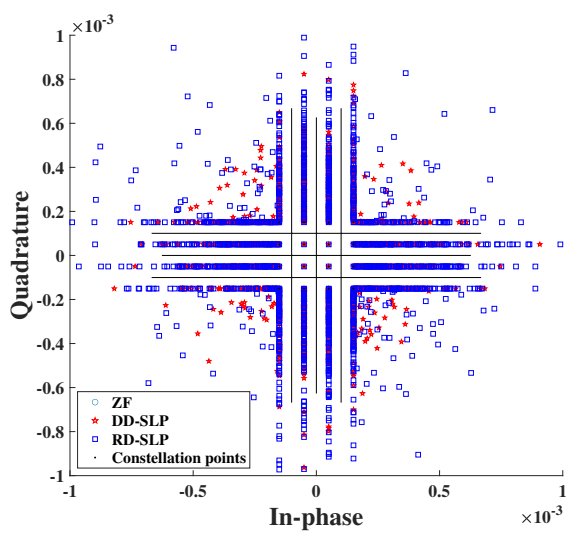

(b)

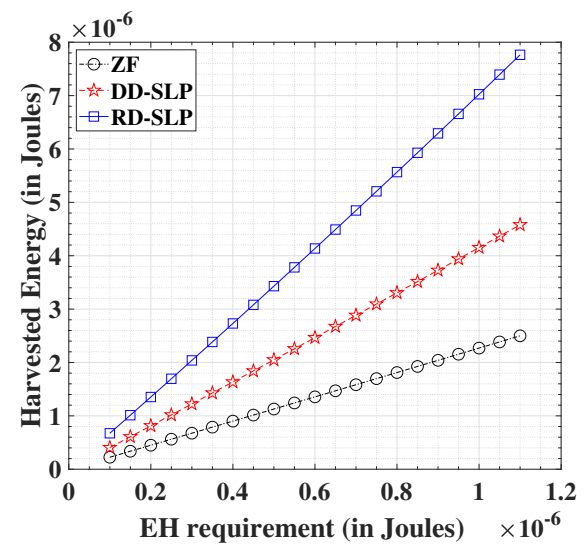

(c)

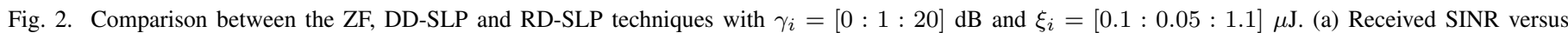

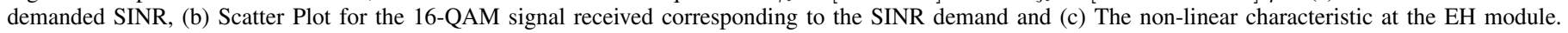

Therefore, the distance preserving CI-based power minimization SLP design for SWIPT boils down to solving the NNLS optimization in (24), which can be tackled via several off-theshelf algorithms; see e.g. [18]-[20].

\section{NumERicAl Results}

To generate numerical results, we consider a MU-MISO system, which has an equal number of the transmit and receive antennas $N_{t}=N_{u}=N$ with $N=64$. We accordingly generate a full rank $N \times N$ MU-MISO channel matrix with i.i.d. complex values according to the ITU-R indoor model ${ }^{5}$ [21].

We average the results over 100 channel matrix iterations, with a defined condition number [13]. We investigate the performances of the proposed methods by measuring the total average power of the precoded symbols generated by the techniques in selected channel scenarios. We set the $\gamma_{i}=[0: 1: 20] \mathrm{dB}$ and $\xi_{i}=[0.1: 0.05: 1.1] \mu \mathrm{J}$ for every $i=1,2, \ldots, N_{u}$. We chose the modulation order $M=16$ to conduct all the experiments. The constants corresponding to the non-linear $\mathrm{EH}$ circuit are chosen as $\mathcal{E}^{\prime}=2.8 \mathrm{~mJ}, \alpha=1500$, and $\beta=0.0022$ [12], [22].

\footnotetext{
${ }^{5} \mathrm{We}$ assume the operational frequency as $915 \mathrm{MHz}$, the separation distance (in metres) between the transmitter and end-user(s) as 5m (i.e., random placement between $1 \mathrm{~m}$ and $5 \mathrm{~m}$ ), and the floor penetration loss factor is chosen to be $9 \mathrm{~dB}$. Transmit antennas are assumed to be directional, with $10 \mathrm{~dB}$ gain.
}

In Fig. 1, we compare the performances of the proposed RDSLP and DD-SLP techniques with the benchmark ZF method. We observe in Fig. 1(a) the increasing patterns of minimized transmit power with an increase in the respective joint SINR and EH demands. On the other hand, we show in Fig. 1(b) and Fig.1(c) the amounts of received signal powers corresponding to the desired SINR and EH operations, respectively, after carrying out the static PS-mechanism (as described in (9)). Herein, the growing SINR and EH demands at the users are seen to be fulfilled by the respectively increasing signal power intensities. Noticeably, the RD-SLP technique outperforms the DD-SLP and $\mathrm{ZF}$ methods corresponding to the chosen investigation parameters. Specifically, we notice an average decrease in the total transmit power via RD-SLP by $161.56 \%$ and via DDSLP by $62.84 \%$ with respect to the ZF method. At the SINR recovering unit, remarkable average gains of $7.82 \%$ and $3.68 \%$ corresponding to the RD-SLP and DD-SLP, respectively, are observed over the ZF. We observe considerable average gains in the harvesting power of $9.48 \%$ and $4.99 \%$ corresponding to the RD-SLP and DD-SLP techniques, respectively, over the ZF method.

In Fig. 2, we compare the performances of the proposed RDSLP and DD-SLP techniques with the benchmark ZF method. We observe in Fig. 2(a) the increasing patterns of the received 
SINR with an increase in the respective SINR demands. We show the corresponding scatter plot for the 16-QAM signal in Fig. 2(b), after carrying out the static PS-mechanism. In Fig. 2(c), we show the non-linear operation at the EH modules of the users where an increase in harvested energy is seen for growing EH demands. Noticeably, the RD-SLP technique outperforms the DD-SLP and ZF methods corresponding to the chosen investigation parameters. Specifically, we notice an increase in the obtained SINR via RD-SLP by $21.63 \%$ and via DD-SLP by $12.07 \%$ with respect to the ZF method. At the EH module, remarkable average performance gains of $101.27 \%$ and $58.12 \%$ corresponding to the RD-SLP and DD-SLP, respectively, are observed over the ZF.

\section{CONCLUSIONS}

In this paper, we presented an SLP based framework to facilitate SWIPT from a single multi-antenna transmitter to multiple single-antenna users. Each user was assumed to employ the PS-mechanism. We formulated the total transmit power minimization problems to investigate three proposed SLP techniques viz., ZF, DD-SLP, and RD-SLP, for SWIPT. The signal design at the transmitter facilitated an easy distribution of the signals received at the users via fixed (static) PS-ratio based on their respective demands of SINR and EH. Such a mechanism did not only reduce an optimization of the additional parameter but also provided tractability to the system from a hardware implementation perspective while guaranteeing maximal gains. Due to the constructive interference and perturbation vectorbased optimizations, tremendous improvements were observed for DD-SLP and RD-SLP over the ZF in terms of received SINR and harvested energy. Additionally, significant benefits of RD-SLP were shown over the DD-SLP and ZF techniques under several test cases.

\section{ACKNOWLEDGMENT}

This research was supported in parts by Luxembourg National Research Fund grant FNR11481283 "End-to-end Signal Processing Algorithms for Precoded Satellite Communications", the FNR-FNRS bilateral project - InWIP-NET : Integrated Wireless Information and Power Networks (R-AGR-0700-10-V), and by the Luxembourg National Research Fund (FNR) under CORE Junior project: C16/IS/11332341 Enhanced Signal Space opTImization for satellite comMunication Systems (ESSTIMS).

\section{REFERENCES}

[1] Y. B. Zikria, S. W. Kim, M. K. Afzal, H. Wang, and M. H. Rehmani, "5G Mobile Services and Scenarios: Challenges and Solutions," Sustainability, vol. 10 , no. 10, 2018.

[2] S. Gautam, et al., "Multigroup Multicast Precoding for Energy Optimization in SWIPT Systems With Heterogeneous Users," IEEE Open Journal of the Communications Society, vol. 1, pp. 92-108, 2020.

[3] S. Gautam, E. Lagunas, S. Chatzinotas, and B. Ottersten, "Wireless multigroup multicast precoding with selective rf energy harvesting," in 2019 27th European Signal Processing Conference (EUSIPCO), A Coruña, Spain, 2019, pp. 1-5.
[4] M. Alodeh, D. Spano, A. Kalantari, C. G. Tsinos, D. Christopoulos, S. Chatzinotas, and B. Ottersten, "Symbol-Level and Multicast Precoding for Multiuser Multiantenna Downlink: A State-of-the-Art, Classification, and Challenges," IEEE Commun. Surveys Tuts., vol. 20, no. 3, pp. 17331757, thirdquarter 2018.

[5] A. Haqiqatnejad, F. Kayhan, and B. Ottersten, "Power minimizer symbollevel precoding: A closed-form suboptimal solution," IEEE Signal Process. Lett., vol. 25, no. 11, pp. 1730-1734, Nov. 2018.

[6] J. Krivochiza, J. Merlano Duncan, S. Andrenacci, S. Chatzinotas, and B. Ottersten, "FPGA Acceleration for Computationally Efficient SymbolLevel Precoding in Multi-User Multi-Antenna Communication Systems," IEEE Access, vol. 7, pp. 15509-15520, 2019.

[7] J. Krivochiza, J. C. Merlano-Duncan, S. Andrenacci, S. Chatzinotas, and B. Ottersten, "Computationally and Energy Efficient Symbol-Level Precoding Communications Demonstrator," Physical Communication, vol. 28, pp. $108-115,2018$.

[8] X. Zhou, R. Zhang, and C. K. Ho, "Wireless Information and Power Transfer: Architecture Design and Rate-Energy Tradeoff," IEEE Trans. Comm., vol. 61, no. 11, pp. 4754-4767, November 2013.

[9] E. Boshkovska, D. W. K. Ng, N. Zlatanov, and R. Schober, "Practical Non-Linear Energy Harvesting Model and Resource Allocation for SWIPT Systems," IEEE Comm. Lett., vol. 19, no. 12, pp. 2082-2085, Dec 2015.

[10] D. W. K. Ng, T. Q. Duong, C. Zhong, and R. Schober, Harnessing Interference in SWIPT Systems, pp. 181-196, Wiley, 2019.

[11] S. Timotheou, G. Zheng, C. Masouros, and I. Krikidis, "Symbol-level precoding in MISO broadcast channels for SWIPT systems," in 2016 23rd Int. Conf. Telecomm. (ICT), Thessaloniki, Greece, May 2016, pp. $1-5$.

[12] J. Guo, H. Zhang, and X. Zhu, "Theoretical analysis of RF-DC conversion efficiency for class-F rectifiers," IEEE Trans. Microw. Theory Techn., vol. 62, no. 4, pp. 977-985, 2014.

[13] J. Krivochiza, J. C. Merlano-Duncan, S. Chatzinotas, and B. Ottersten, "M-QAM Modulation Symbol-Level Precoding for Power Minimization: Closed-Form Solution," in 2019 16th International Symposium on Wireless Communication Systems (ISWCS), Oulu, Finland, Aug 2019, pp. 395-399.

[14] S. Gautam, E. Lagunas, S. Vuppala, S. Chatzinotas, and B. Ottersten, "Pricing Perspective for SWIPT in OFDM-based Multi-User Wireless Cooperative Systems," in 2019 IEEE Wireless Communications and Networking Conference (WCNC), Marrakesh, Morocco, April 2019, pp. $1-7$.

[15] M. Grant and S. Boyd, "CVX: Matlab Software for Disciplined Convex Programming, version 2.1," http://cvxr.com/cvx, Mar. 2014.

[16] A. Haqiqatnejad, F. Kayhan, and B. Ottersten, "Constructive interference for generic constellations," IEEE Signal Process. Lett., vol. 25, no. 4, pp. 586-590, Apr. 2018.

[17] A. Haqiqatnejad, F. Kayhan, and B. Ottersten, "Symbol-level precoding design based on distance preserving constructive interference regions," IEEE Trans. Signal Process., vol. 66, no. 22, pp. 5817-5832, Nov. 2018.

[18] R. Bro and S. De Jong, "A fast non-negativity-constrained least squares algorithm," Journal of Chemometrics: A Journal of the Chemometrics Society, vol. 11, no. 5, pp. 393-401, 1997.

[19] Roman A Polyak, "Projected gradient method for non-negative least square," Contemp Math, vol. 636, pp. 167-179, 2015.

[20] Yurii E Nesterov, "A method for solving the convex programming problem with convergence rate $\mathrm{O}\left(1 / \mathrm{k}^{\wedge} 2\right)$," in Dokl. Akad. Nauk SSSR, 1983, vol. 269, pp. 543-547.

[21] P. Series, "Propagation data and prediction methods for the planning of indoor radiocommunication systems and radio local area networks in the frequency range $900 \mathrm{MHz}$ to $100 \mathrm{GHz}$," Recommendation ITU-R, pp. 1238-7, 2012.

[22] S. Gautam, E. Lagunas, S. Chatzinotas, and B. Ottersten, "Relay Selection and Resource Allocation for SWIPT in Multi-User OFDMA Systems," IEEE Trans. Wireless Commun., vol. 18, no. 5, pp. 2493-2508, May 2019. 